\title{
Clinical value of Doppler echocardiography in the assessment of adults with aortic stenosis
}

\author{
IAIN A SIMPSON, ALAN B HOUSTON, CHRIS D SHELDON, IAN HUTTON, \\ T D V LAWRIE \\ From the University Department of Medical Cardiology, Royal Infirmary, Glasgow
}

SUMmaRy Continuous wave Doppler echocardiography was used to study 41 adults with clinically suspected aortic stenosis undergoing cardiac catheterisation. Non-invasive assessment of the severity of stenosis was made before catheterisation using electrocardiograms, chest radiographs, and cross sectional echocardiography in addition to clinical examination and assessment modified, where appropriate, by the result of the Doppler examination. Catheterisation gradients were obtained in 33 patients and correlated well with those obtained by Doppler examination particularly when simultaneous recordings were obtained. All patients with surgically significant stenoses were identified by non-invasive assessment including Doppler examination and overestimation was not found in any patient with a less than significant stenosis.

Thus surgery can be recommended in patients with aortic stenosis without the need for previous cardiac catheterisation.

Cross sectional echocardiography is a well established non-invasive method of assessing the severity of valvar stenoses. It is of particular value in the assessment of mitral stenosis, ${ }^{1}$ but difficulties can arise in accurately assessing patients with aortic stenosis. ${ }^{2} 3$

The ability of Doppler echocardiography to measure transvalvar gradients has obvious potential benefits in the non-invasive assessment of patients with aortic stenosis. Pulsed wave Doppler ultrasound has been used to measure blood flow velocity in the ascending aorta ${ }^{4}$ and to establish normal values. ${ }^{5}$ Nevertheless, it is unable to measure the high velocities present in patients with aortic stenosis, a disadvantage not found with the continuous wave Doppler technique. Using the continuous wave Doppler technique recent reports have suggested that in patients with aortic stenosis there is a good correlation between the Doppler derived gradient and that obtained at catheterisation, ${ }^{6-8}$ but the potential clinical application of the technique has yet to be fully established. This study was therefore undertaken in patients with clinically suspected aortic stenosis to confirm the accuracy of continuous wave Doppler ultrasound in predicting valve gradients and in par-

Requests for reprints to Dr I A Simpson, University Department of Medical Cardiology, Royal Infirmary, Glasgow G31 2ER.

Accepted for publication 31 January 1985 ticular to assess its clinical value and possible role in the subsequent management of these patients.

\section{Patients and methods}

The study group comprised 41 adults (age range 14-73 (mean 52) years) undergoing cardiac catheterisation with clinically suspected aortic stenosis either alone or in combination with other valve lesions. All patients had clinical examination performed by two experienced cardiologists. In addition a 12 lead electrocardiogram, chest radiograph, and cross sectional echocardiogram were performed in all patients before cardiac catheterisation.

\section{DOPPLER ECHOCARDIOGRAPHY}

Doppler examination was performed using a Vingmed Alfred continuous and pulsed wave velocimeter with a $2 \mathrm{MHz}$ transducer. Continuous wave velocimetry was used for all examinations to obviate the effects of aliasing occurring in the measurement of high velocities in the pulsed mode. Being both gain and threshold dependent there is a tendency for the analogue display of maximum velocity to underestimate valve gradient, ${ }^{6}$ and so spectrum analysis was used in all cases, using either an Angioscan or Doptek analyser. Doppler examination was performed in the resting state from a variety of precordial positions in order to 
obtain the Doppler signal of maximum systolic blood flow velocity in the ascending aorta. The audio signal was used in addition to spectrum analysis to obtain the Doppler signal indicating laminar flow and the maximum systolic frequency shift then obtained from a clearly demarcated spectral signal without obvious interference (Fig. 1). In an attempt to minimise the risk of falsely low recordings all possible precordial positions were examined including suprasternal, apical, subcostal, supraclavicular, and right parasternal in the right lateral decubitus position.

The maximum systolic velocity obtained in the ascending aorta by the Doppler examination was used to calculate the transvalvar gradient using a modification of the Bernoulli formula; $P=4 V^{2}$ where $P$ is the transvalvar gradient $(\mathrm{mm} \mathrm{Hg}$ ) and $\mathrm{V}$ the maximum systolic velocity (metres $\mathrm{s}^{-1}$ ). Before cardiac catheterisation an assessment of the severity of aortic obstruction was made by the attending physician using clinical examination with the addition of an electrocardiogram, chest radiograph, and cross sectional echocardiogram. This was graded as either non-significant, surgically significant, or severity in doubt from non-invasive information. The result of the Doppler examination was then added and any appropriate modification of the assessment of severity made.

\section{CARDIAC CATHETERISATION}

All patients underwent full cardiac catheterisation using Judkins's technique and where possible peak to peak systolic gradients (in $\mathrm{mm} \mathrm{Hg}$ ) obtained by withdrawal of a fluid filled multihole catheter across the aortic valve. In addition two patients underwent

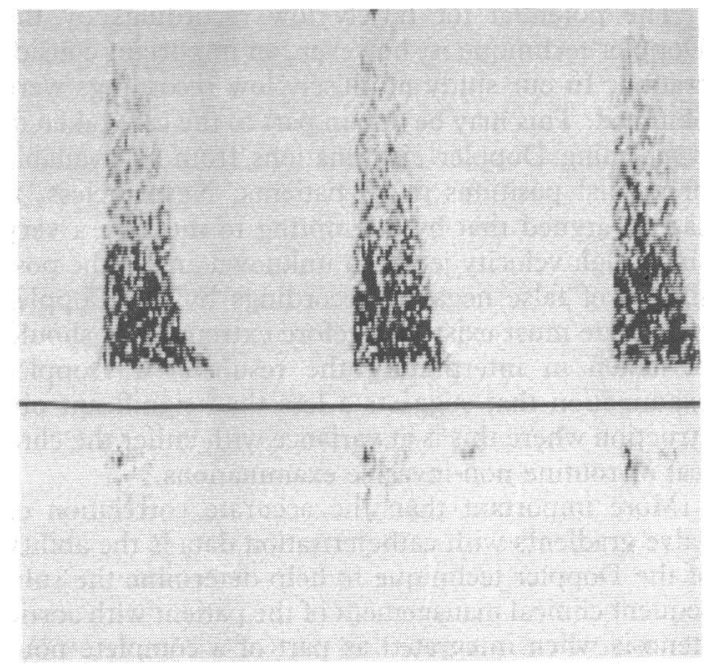

Fig. 1 Continuous wave Doppler echocardiogram from the suprasternal notch indicating a clearly demarcated spectral signal in a patient with an aortic valve gradient of $37 \mathrm{~mm} \mathrm{Hg}$. transseptal catheterisation using the Brockenbrough technique in one and via a patent foramen ovale in another. All patients were prescribed $10 \mathrm{mg}$ diazepam orally as a premedication 30 minutes before catheterisation. This may have the effect of lowering cardiac output and transvalvar gradient. In an attempt to assess the effects of sedation on the aortic valve gradient 24 of the 33 patients had a further Doppler examination performed at the time of cardiac catheterisation immediately after withdrawal of the catheter across the aortic valve.

The decision regarding subsequent patient management was made by the attending physician on the basis of all available clinical and non-invasive data in addition to the results of cardiac catheterisation, and a comparison could then be made with the decision reached by non-invasive techniques alone.

\section{Results}

Satisfactory Doppler signals were obtained in all patients. Though the suprasternal notch was the most successful position for obtaining the optimum Doppler signal (29 of 41 patients) it was not possible to predict the most satisfactory precordial examination position even when cross sectional echocardiography was used. Indeed, although several positions were usually successful in each patient, the right parasternal position was most successful in six, the subcostal in five, and the apical in one, and in one patient the only satisfactory view was obtained from the right supraclavicular position.

In eight of the 41 patients undergoing cardiac catheterisation it was not possible to cross the aortic valve retrogradely. Transseptal catheterisation was not undertaken at the discretion of the attending physician and therefore no gradients were obtained in these patients.

In the remaining 33 patients the catheter gradients ranged from 0 to $120 \mathrm{~mm} \mathrm{Hg}$ with a mean of $43 \mathrm{~mm}$ $\mathrm{Hg}$ and the Doppler gradients from 8 to $157 \mathrm{~mm} \mathrm{Hg}$ with a mean of $49 \mathrm{~mm} \mathrm{Hg}$. Figure 2a shows the overall correlation between the catheter derived gradients and those obtained by Doppler. In addition, the correlation between the Doppler derived gradients obtained simultaneously at catheterisation and the catheter gradients is shown (Fig. 2b). A superior correlation $(r=0.98)$ was obtained by this method, presumably because of the effects of sedation. This is suggested by the fact that in the majority (70\%) Doppler gradients obtained simultaneously at catheterisation were lower than those obtained without the effects of sedation.

In the 33 patients in whom valve gradients were subsequently obtained at catheterisation the noninvasive assessments with and without Doppler meas- 
urements were compared with the subsequent outcome from the complete data including that obtained at catheterisation. Though in general terms a peak aortic gradient of $>50 \mathrm{~mm} \mathrm{Hg}$ was taken as surgically significant in one patient the gradient at Doppler examination was $42 \mathrm{~mm} \mathrm{Hg}$ and $45 \mathrm{~mm} \mathrm{Hg}$ at catheterisation. This was regarded as significant by the attending physician and therefore analysed as such.

In the nine patients with clinically non-significant lesions the Doppler findings suggested a nonsignificant lesion in seven, which was subsequently confirmed by catheterisation. In the remaining two patients, however, Doppler examination suggested a surgically significant lesion, which was confirmed by catheterisation in both cases.

In the 12 patients thought clinically to have surgically significant aortic stenosis Doppler examination identified a surgically significant gradient in all 12 , which was subsequently confirmed by catheterisation. The remaining 12 patients, however, had inconclusive evidence on clinical and non-invasive data. In 10 patients Doppler examination could not identify a

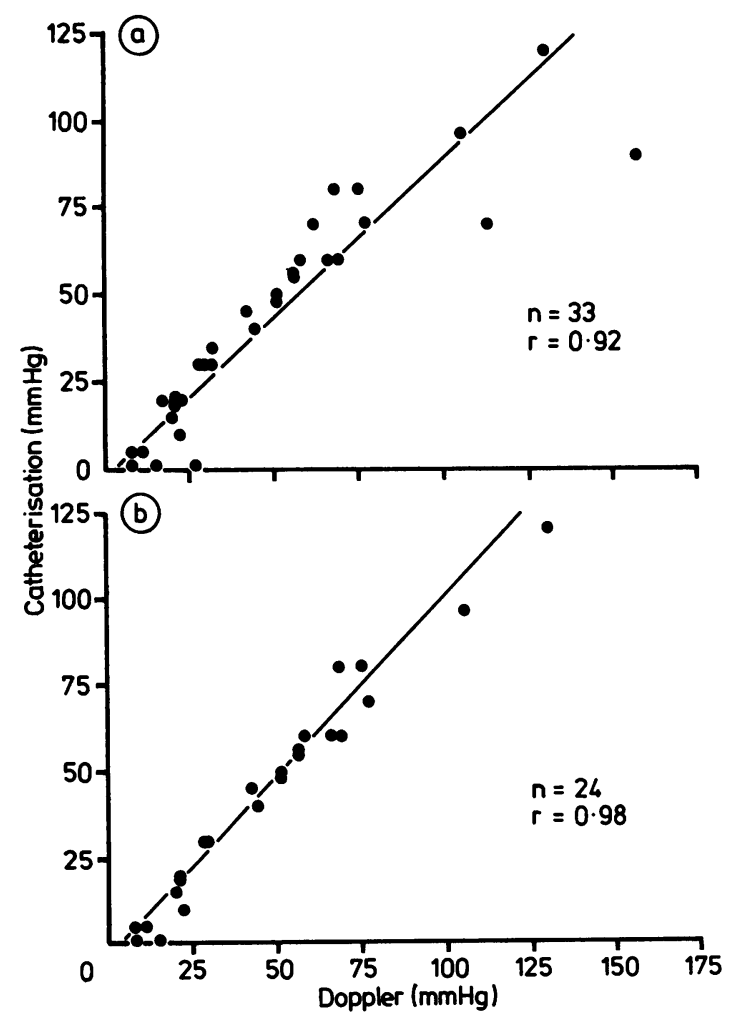

Fig. 2 Correlation of valve gradients obtained using the continuous wave Doppler technique and (a) those subsequently found at cardiac catheterisation and $(b)$ those obtained at simultaneous cardiac catheterisation. significant gradient nor did subsequent catheterisation. The two surgically significant lesions detected by the Doppler technique were, however, confirmed by catheterisation.

\section{Discussion}

It has been suggested that the non-invasive assessment of valve disease can obviate the need for cardiac catheterisation in many cases. ${ }^{10}$ Patients with aortic stenosis are, however, often the most difficult to assess non-invasively, and cardiac catheterisation is almost always required. In this study, however, in an appreciable number of patients (eight of 41) it was not possible even at cardiac catheterisation to obtain a valve gradient. In comparison satisfactory signals were recorded in all patients investigated by the Doppler technique.

The correlation found between the Doppler derived gradient and that obtained at catheterisation indicates that Doppler echocardiography provides an accurate assessment of the gradient across an aortic obstruction. This measurement did not appear to be appreciably affected by the fact that peak to peak systolic gradients were measured at catheterisation rather than an instantaneous value as measured by the Doppler technique. ${ }^{11}$ In several cases the sedative used as a premedication for catheterisation affected the Doppler derived gradient, slightly lowering its value compared with previous Doppler examinations. This probably accounts for the better correlation between simultaneous recordings and also for the less accurate correlations reported in other studies where simultaneous measurements were not made. ${ }^{89}$

The potential for falsely low recordings by the Doppler technique is, however, an important consideration. In our study no falsely low recordings were obtained. This may be due in part to the care taken in performing Doppler examinations from all available precordial positions in all patients. Nevertheless, it can be argued that by attempting to measure a very small high velocity jet at an unknown angle, the possibility of false negative recordings by the Doppler technique must exist. Therefore extreme care should be taken in interpreting the result of a Doppler examination that suggests a less than significant obstruction where this is at variance with either the clinical or routine non-invasive examinations.

More important than the accurate correlation of valve gradients with catheterisation data is the ability of the Doppler technique to help determine the subsequent clinical management of the patient with aortic stenosis when integrated as part of a complete noninvasive assessment. Indeed in our study no patient with a surgically significant lesion would have been missed by complete non-invasive assessment includ- 
ing Doppler examination, and in no patient was there an appreciable overestimation of the severity of obstruction. Where doubt existed as to the severity of a lesion by other non-invasive techniques alone, Doppler examination was able to correctly clarify the degree of obstruction in all cases. Possibly more important is the fact that the Doppler examination identified two patients with surgically significant lesions that would otherwise have been missed by routine clinical and non-invasive assessment.

It can be argued that cardiac catheterisation may be indicated for other valve lesions, but, as previously reported, the vast majority of these can be accurately assessed by standard non-invasive techniques. ${ }^{10}$ Where the possibility of ischaemic heart disease exists coronary angiography may be indicated, but this is a much less hazardous and time consuming procedure than full right and left heart cartheterisation. Indeed in an appreciable number of patients undergoing catheterisation for valvar disease coronary angiography is not performed. ${ }^{10}$

Doppler echocardiography has therefore added a new dimension to the assessment of patients with aortic stenosis. When combined with clinical examination and other routine non-invasive techniques it can accurately determine the subsequent clinical management of these patients, and it would therefore seem safe and appropriate to recommend surgery in an appreciable number of patients with aortic stenosis without the need for previous cardiac catheterisation.

\section{References}

1 Martin RP, Rakowski H, Kleiman JH, Beaver W, Lon- don E, Popp RL. Reliability and reproducibility of twodimensional echocardiographic measurement of the stenotic mitral valve orifice area. Am f Cardiol 1979; 43: 560-8.

2 Godley RW, Green D, Dillon JC, Rogers EW, Feigenbaum H, Weyman AE. Reliability of two-dimensional echocardiography in assessing the severity of valvular aortic stenosis. Chest 1981; 79: 657-62.

3 DeMaria AN, Bommer W, Joye J, Lee G, Bouteller J, Mason DT. Value and limitations of cross-sectional echocardiography of the aortic valve in the diagnosis and quantification of valvular aortic stenosis. Circulation 1980; 62: 304-12.

4 Angelsen BAJ, Brubakk AO. Transcutaneous measurement of blood flow velocity in the human aorta. Cardiovasc Res 1976; 10: 368-79.

5 Gardin JH, Burn CS, Childs WJ, Henry WL. Evaluation of blood flow velocity in the ascending aorta and main pulmonary artery of normal subjects by Doppler echocardiography. Am Heart f 1984; 107: 310-9.

6 Hatle L, Angelsen BA, Tromsdal A. Non-invasive assessment of aortic stenosis by Doppler ultrasound. $\mathrm{Br}$ Heart f 1980; 43: 284-92.

7 Stamm RB, Martin RP. Quantification of pressure gradients across stenotic valves by Doppler ultrasound. $\mathcal{F}$ Am Coll Cardiol 1983; 2: 707-18.

8 Berger M, Berdoff RL, Gallerstein PE, Goldberg E. Evaluation of aortic stenosis by continuous wave Doppler ultrasound. f Am Coll Cardiol 1984; 3: 150-6.

9 Hatle L, Brubakk A, Tromsdal A, Angelsen B. Noninvasive assessment of pressure drop in mitral stenosis by Doppler ultrasound. Br Heart f 1978; 40: 131-40.

10 Hall RJC, Kadushi OA, Evemy K. Need for cardiac catheterisation in assessment of patients for valve surgery. Br Heart $\mathcal{F}$ 1983; 49: 268-75.

11 Hatle L, Angelsen B. Doppler ultrasound in cardiology. Philadelphia: Lea and Febiger, 1982: 92. 